\title{
Ejercicio exploratorio de comparabilidad de las prácticas de discriminación: los casos de la Argentina y Chile
}

\section{Exploratory exercise on comparability of discrimination practices: the cases of Argentina and Chile}

\author{
Pablo Molina Derteano \\ pablomd2009@gmail.com \\ Instituto de Investigaciones Gino \\ Germani. CONICET - Universidad de \\ Buenos Aires, Argentina
}

Recepción: 06 Junio 2020

Aprobación: 07 Noviembre 2020

Publicación: 01 Febrero 2021

Cita sugerida: Molina Derteano, P. (2021). Ejercicio exploratorio de comparabilidad de las prácticas de discriminación: los casos de la Argentina y Chile. Cuestiones de Sociología, 24, e111. https://doi.org/10.24215/23468904e111
Resumen: El presente artículo se propone explorar las relaciones entre un enfoque radical de la desigualdad que considera las posiciones estructurales y las prácticas de la discriminación. Revisando la literatura reciente, en especial en discriminación estructural, se propone un modelo explicativo en el que se comparan los casos de la Argentina y Chile con datos del Mapa Nacional de la Discriminación (Segunda Edición) del primero y el módulo en la encuesta CASEN en el segundo. Se miden por separado la incidencia de las posiciones socioeconómicas a partir del máximo nivel educativo del Principal Sostén de Hogar (Modelo 1) y la incidencia del género, el origen migrante y la pertenencia a pueblos originarios (Modelo 2) en la experiencia de haber sufrido algún tipo de discriminación. En ambos modelos, las variables independientes se muestran significativas, aun cuando la bondad de ajuste es modesta. El Modelo 1 es más robusto en el caso argentino.

Palabras clave: Discriminación sufrida, Posiciones estructurales, Comparación.

\begin{abstract}
This article aims to explore the relationships between a radical approach to inequality that considers the structural positions and the practices of discrimination. Exploring the recent literature, especially in structural discrimination, an explanatory model is proposed and the cases of Argentina and Chile are compared with data from the National Map of Discrimination (Second Edition) of the first and the module in the CASEN survey in the second. The incidence of socioeconomic positions from the highest educational level of the Main Home Support (Model 1) and the incidence of gender, migrant origin and belonging to native peoples (Model 2) in the experience of having suffered some type of discrimination are measure separately. In both models, the independent variables are significant, even when the goodness of fit is modest. Model 1 is more robust in the Argentine case.
\end{abstract}

Keywords: Suffered Discrimination, Structural positions, Comparison. 


\section{Introducción}

En la miniserie para televisión "Gambito de Dama” ("The Queen's Gambit", 2020) la protagonista Elizabeth Harmon intenta triunfar en el mundo del ajedrez superando prejuicios institucionalizados por su condición de mujer y sus conductas adictivas. En el último episodio de la miniserie, Harmon va a competir a la URSS y se resalta que se trata de una campeona femenina, que compite contra hombres. Según el narrador, estaría presente, entre el público, otra campeona, Nona Gaprindashvili, quien "nunca habría competido contra varones". En el caso de Harmon, ella podría reconocer y dar cuenta de la discriminación sufrida por su condición de mujer, mientras que Nona estaría inmersa en una trama de discriminación estructural que no sería tan evidente.

La discriminación como problema social y académico, y como objeto de políticas públicas, ha estado presente en la agenda de muchos Estados nacionales desde mediados del siglo XX. Incluso hay cierto statu quo internacional en condenar su práctica y desarrollar estrategias para su prevención (Kauffman, 1991; Solís, 2017). El importante cúmulo de evidencias y debates resaltan, entre otras cuestiones, ciertas tensiones en las operaciones de definición e identificación de la discriminación. El presente artículo se propone 1) explorar en clave teórica y comparativa la definición de discriminación estructural y sus tensiones con las definiciones radicales de desigualdad, así como las dificultades para su identificación; 2) indagar críticamente sobre las formas de medir en forma directa la discriminación como experiencia sufrida, comparando los casos de Chile y la Argentina.

\section{Definiciones y desafíos en la medición}

Según el Instituto Nacional contra la Discriminación, el Racismo y la Xenofobia (INADI), la discriminación es la práctica de "marginar a una persona o grupo de personas, en relación con determinadas características, como el género, sus creencias religiosas o politicas, su nacionalidad, su situación social o económica, su orientación sexual, su edad, su pertenencia a un pueblo indigena, sus caracteristicas fisicas, por las cuales se lo trata como inferior, negando sus derechos y oportunidades." $(2013,12)$. Solís la define como "el conjunto de prácticas, informales o institucionalizadas, que niegan el trato igualitario o producen resultados desiguales para ciertos grupos sociales y que tienen como consecuencias la privación o el menoscabo en el acceso a los derechos y la reproducción de la desigualdad social." $(2017,27)$. Ambas definiciones coinciden en que se niega un trato igualitario con diferentes niveles de daño; lo que supone que, en términos formales, existiría un principio de igualdad que no se estaría respetando. El segundo componente es que la persona o el grupo discriminado pertenece a un colectivo que, indirectamente, es objeto del menoscabo. Por tanto, para que se dé una práctica discriminatoria, se debe primeramente atribuir a un colectivo una identidad inferior y/o peligrosa; y luego, atribuir a un grupo o persona pertenencia a tal colectivo. En el ejemplo de ficción que inicialmente se presentó, Elizabeth es menospreciada por su pertenencia al colectivo "mujeres", el cual es calificado como "inferior" en el marco de sociedades patriarcales y en el ámbito del ajedrez profesional. 
Llegado a este punto, resulta de interés destacar el rol que juega el sistema de posiciones -o campo en términos bourdieanos- en el que se inscriben las prácticas discriminatorias. Este será el foco de nuestra indagación: un análisis de la discriminación estructural como un sistema en sí mismo (Pincus, 1996; Molina Derteano, en prensa) Una parte importante de la literatura sobre la temática ha centrado el análisis en el conjunto de prejuicios que definen a los grupos subalternos y/o peligrosos (Cohen, 2009), mientras otra parte se enfoca en la percepción de de dichos grupos sobre la discriminación sufrida y la discriminación percibida (INADI, 2014)

Esta primera distinción es una de las múltiples que se podría aplicar, pero en este artículo se intentará abordar dos líneas de problematización acerca de la discriminación cómo práctica social.

\section{La discriminación y la desigualdad socioeconómica}

El punto de partida para esta línea de indagación es el trabajo pionero de Gary Becker, de 1957, “ he economics of Discrimination”, que más allá de los análisis económicos, consideraba el efecto del prejuicio en detrimento del capital humano, en la selección de trabajadores por parte de los empleadores (Leblanc, 1995; Kerwin y Guryan, 2007). Este trabajo condenaba los prejuicios no sólo desde un punto de vista moral, sino también por sus consecuencias económicas ya que los prejuicios individuales de los empleadores no les permitían contar con la mejor mano de obra. Arrow (1998), por su parte, demuestra la vigencia de los prejuicios a un nivel tal que aquellos perjudicados por ellos tienden a no querer incrementar su capital humano, creyendo que no serán valorados.

La visión liberal ha contribuido a conceptualizar los prejuicios como coordenadas de análisis extraeconómicas vinculadas a las construcciones culturales (Montes Berges 2008; Wagram, 2012), el racismo (Bobo y Fox, 2003; Wieviorka, 2007; Reskin, 2012) y la xenofobia (Cea D'Ancona y Valles, 2015). Estos conceptos ocupan un campo de estudios en sí mismos, y son operacionalizados como factores intervinientes externos a las asimetrías en las posiciones resultantes del mercado de trabajo (Pincus, 1996). En términos weberianos, las coordenadas de etnia y xenofobia, así como el género, son formas de estatus que componen grupos o agrupamientos sociales, con sus propias formas de asimetría (Molina Derteano, en prensa).

Esta denominada tradición liberal considera las desigualdades de posiciones socioeconómicas o socio-ocupacionales como resultantes de las diferencias de mérito o de capital humano (Mora Salas, 2004; Molina Derteano, op. cit.), mientras que las asimetrías de género, etnia, nacionalidad, raza o de otro tipo son factores intervinientes "externos", que se ponen en juego en la interacción entre actores individuales.

Desde otra corriente, se pueden agrupar los aportes teóricos y metodológicos en el foco sobre la desigualdad de posiciones que crearían las condiciones para el cierre social. Los estudios sobre desigualdad de posiciones tienen muchos puntos de contacto con la perspectiva radical de la desigualdad (Mora Salas, op. cit.). Para esta perspectiva, la discriminación estructural es el objeto de estudio y se lo define por su continuidad en el tiempo y su significación estadística. Así, los motivos de la discriminación y los grupos específicos que resultan afectados pasan 
a un segundo plano, pues el foco estaría en las relaciones entre la discriminación como mecanismo de cierre social y la incidencia de las clases sociales, el género y/o el racismo desde un punto de vista sistémico e interseccional (Browne y Misra, 2003; Romero, 2017). Raza, género y clase social refieren a lógicas sistémicas que "explican y/o justifican" la desigual distribución de posiciones de estatus de los sujetos y, desde ese punto de partida, se analiza la discriminación estructural como mecanismo de cierre social. Conceptos como las consecuencias estructurales de las prácticas discriminatorias (Solís, op. cit.), la racialización de las relaciones de clase (Margulis, 1999) o la matriz racista de la desigualdad (INADI, op. cit.) son aportes a un intento de considerar las diversas formas y motivos de las prácticas discriminatorias en articulación con las desigualdades de clase resultantes de la sociedad capitalista.

En un nivel más abstracto, se ubican las propuestas metodológicas del análisis de la discriminación múltiple (Cea D’Ancona, 2005; 2019), así como el concepto de interseccionalidad, que da cuenta de la confluencia de dos o más coordenadas de desigualdad, lo que produce un efecto singular sui géneris, que no debería ser separado analíticamente (Browne y Misra, op. cit.; Romero, op. cit.). Estas propuestas dan cuenta de que difícilmente pueda abordarse el estudio de coordenadas de género o de etnia en forma separada entre sí y/o mediadas por las clases sociales.

En este sentido, este artículo se puede inscribir en un programa de investigación más amplio: explorar en qué medida determinadas clases ocupacionales se encuentran estadísticamente más propensas a sufrir prácticas discriminatorias, relegando a un segundo lugar analítico los motivos y las formas de tales prácticas.

\section{La discriminación y su percepción}

Para algunos autores, sobre todo provenientes de campos relacionados con la psicología y con la psicología social, el registro de las percepciones sobre la discriminación es complejo. Canache y otros (2014) afirman que no se cuenta con un medio de registro robusto y no hay certeza de que las discriminaciones sufridas sean percibidas como tales. Bajo esta lógica, las prácticas discriminatorias son concebidas como "reactualizaciones" de posiciones diferenciales. Volviendo al ejemplo de ficción, Elizabeth puede dar cuenta de la discriminación sufrida porque intenta introducirse en un campo muy masculinizado (el del ajedrez profesional), mientras que Nona se desempeña en el del ajedrez femenino, que resulta de la misma lógica patriarcal pero de una forma en la que la transgresión es menos posible. Cuando Elizabeth intenta hacerse un nombre en un espacio en donde predominan los varones, las prácticas discriminatorias surgen para "ponerla de vuelta en su sitio", que sería el de ama de casa y madre y nada más.

En la segunda mitad del siglo XX, se fueron acumulando iniciativas nacionales e internacionales destinadas a prevenir y sancionar las prácticas discriminatorias, incluyendo "condenas públicas" por expresiones en las redes sociales y en los medios de comunicación. Cabe indagar si, efectivamente, quienes discriminan explicitan sus motivos arriesgándose a sanciones, pero también si quienes sufren prácticas de discriminación pueden dar cuenta de su pertenencia a un grupo segregado, o tan siquiera, si ese grupo al que pertenecen puede ser identificado 
como un grupo segregado (Kaufman, 2010; Barbera, 2011; Canache et al. 2103; Nuvaez Castillo, 2019). Esta dificultad se manifiesta en dos niveles.

En un primer nivel puede ocurrir que las desigualdades estén tan naturalizadas que la discriminación no sea advertida (Cea D’Ancona, 2005; Valles, 2015; Allen y Cutts, 2019; Montoya, Parrado, Solís y Undurranga) o que se dé la paradoja de que quienes más la sufren en términos de clase social no cuenten con el capital cultural para reconocerlas como tales (Molina Derteano, 2019). Otros factores de tipo histórico o cultural pueden estar actuando (Grimson, 1997; 2006; y Denardi, 2016; Birzer y Ellis, 2006). Para medir esta dificultad en percibir y/ o verbalizar las prácticas discriminatorias son necesarias estrategias cualitativas (Chávez Molina y Molina Derteano, 2018; Solís Krozer, Batista y Graniel, 2019) o bien diseños experimentales, con base en la disciplina de la psicología social (Bobo y Fox, 2003; Canache et al., op. cit.).

En un segundo nivel, porque puede tratarse de formas de discriminación indirecta (Barbera, op. cit.; MSSI,2012) o formas de discriminación institucional y/o estructural (Pincus, op. cit.; Solís, op. cit.), cuyo funcionamiento tiende a presentarse despersonalizado y no resulta fácilmente identificable como práctica de discriminación por quienes la sufren o atestiguan. En este sentido, un segundo objetivo tiene que ver con explorar el grado de reconocimiento de las prácticas de discriminación por mujeres, descendientes de pueblos originarios y migrantes extranjeros, que serían grupos especialmente afectados.

Como resumen de lo hasta aquí expuesto, puede decirse que este artículo ha revisado algunos aportes sobre la literatura internacional en torno a la discriminación, y por tanto:

- Se cuenta con una definición operativa por la cual la discriminación es una práctica de marginación, agresión y/o negación de derechos hacia una persona o grupo de personas en virtud de su pertenencia a un colectivo que es definido como subalterno y/o peligroso.

- Puede tenderse una asociación entre los estudios de desigualdad y las prácticas de discriminación por cuanto ambas plantean un espacio social con un conjunto de posiciones relaciones y asimétricas entre sí. La discriminación es una práctica asociada a la prevención de movimientos entre posiciones considerados como ilegítimos por un orden social dominantes. Esta ilegitimidad puede ser construida tanto por el movimiento en sí como por quien lo realiza o por la forma en que lo realiza.

- De entre los varios aspectos de la literatura en torno al fenómeno de la discriminación, el racismo y la xenofobia, se hace un recorte en torno al punto de vista liberal, que define las coordenadas de género, etnia, raza y/o nacionalidad, entre otras, como ajenas a la distribución legitimada, que sería la resultante del mercado, la meritocracia y la pugna entre capitales (económicos, sociales y culturales). Este aspecto de interacción es matizado aquí, proponiendo el estudio de la mayor tendencia a ser discriminado por motivos de raza, etnia y/o género de aquellos individuos pertenecientes a los hogares con menores dotes de capital u otro criterio de estratificación.

- Finalmente, se rescatan algunos aportes sobre la opacidad de la práctica discriminatoria y su percepción por quienes la sufren, en especial cuando 
las prácticas discriminatorias asumen la forma indirecta, institucional y/ o estructural que suelen presentarse como despersonalizada.

Considerando estos aportes, se plantea un ejercicio exploratorio comparando los factores que modifican las chances de sufrir discriminación para los casos de Chile y la Argentina.

\section{Propuesta metodológica}

Desde la segunda mitad del siglo XX, hubo una proliferación de estudios sobre discriminación en el espectro noratlántico que apelaron a la utilización de datos agregados para medir la ocurrencia de prácticas discriminatorias, así como las percepciones y creencias de las poblaciones que pudieran llegar a causarlas y/ o legitimarlas. Si se consideran estudios clásicos como los de heodor Adorno, estos estudios siempre han enfrentado el llamado sesgo de "deseabilidad social" (Cea D’Ancona, 2012). Por este, se reconoce la dificultad de que los respondientes de una metodología de encuesta reconozcan que incurren en prácticas discriminatorias o inclusive que las sufren o hayan sufrido. Este último aspecto se ha visto suavizado en los últimos años, a pesar de que las interrogaciones directas pueden tener menos eficacia que las estrategias cualitativas o las preguntas por medio de escalas de opinión, que son más comprensivistas.

Dado que este artículo tiene un alcance exploratorio y descriptivo, se tomarán dos relevamientos para los casos argentino y chileno, para comenzar a explorar el análisis de la discriminación estructural por posiciones y la incidencia de los factores asociados a la etnia, el género y la nacionalidad. Para el caso argentino se toman datos de un relevamiento específico sobre discriminación conocido como el Mapa Nacional de la Discriminación, realizado en 2013; mientras que para Chile se toman los datos de la Encuesta de Caracterización Socioeconómica Nacional ( en adelante, CASEN), que en 2017 incluyó un módulo destinado al relevamiento de la problemática de la discriminación. Ambas fuentes de datos presentan importantes diferencias, que han sido armonizadas de cara a la posible comparabilidad.

En primer lugar, debe señalarse que ambos países cuentan con una tradición importante de estudios sobre la problemática de la discriminación y que incluyen políticas a nivel legislativo: la ley 23592/88, denominada Ley de ejercicio de derechos y garantías constitucionales y medidas contra actos discriminatorios en el caso argentino, y la ley 20609, para el caso chileno y que fue sancionada en 2012. Se la conoce como ley antidiscriminatoria o, coloquialmente, como "Ley Zamudio". Ambas leyes facultan, entre otras cuestiones, sanciones para quienes cometan actos de discriminación. Nótese la importante distancia cronológica entre ambos países en contar con estos instrumentos legales, que es atribuible a los diferentes ritmos con que fueron dejando atrás los regímenes autoritarios de la década de los 70 y sus reminiscencias. Mientras que, en el caso argentino se suceden una serie de leyes aperturistas en la década de los 80 , el proceso chileno es más lento. Excede el espacio de este artículo hacer una revisión de los sucesos aperturistas en cada país, y sus diferentes valoraciones, pero puede usarse una comparación ilustrativa. Los datos que se utilizarán de la Argentina fueron 
construidos 25 años después de la sanción de la ley antidiscriminatoria de ese país; los datos de Chile, sólo 5 años después. Tampoco se revisará en extenso la literatura referida a los antecedentes de investigación sobre la temática, pero cabe destacar que, a pesar de que la ley antidiscriminatoria es más reciente, en Chile los estudios sobre discriminación y, en especial, referentes a la etnia mapuche tienen una larga trayectoria muy anterior a la Ley Zamudio (Barandian, 2012). En el relevamiento argentino, un 33,2 \% admite haber sufrido discriminación, mientras que en el caso chileno llega a un 14,3\%. Dicha diferencia podría explicarse, en parte, en la diferencia cronológica del tema en la agenda pública de ambos países.

La Segunda Encuesta Nacional de Discriminación fue realizada en 2013 y relevó un total de 14.800 casos, para un universo de 25.951 .593 casos de adultos de 18 años y más. El muestreo fue probabilístico, multietápico y estratificado con selección aleatoria de unidades primarias. El relevamiento fue organizado por provincia, distinguiendo entre área capital y municipios y ciudades del interior de cada provincia, con la excepción del caso de las provincias de Santa Fe y Buenos Aires, que fueron divididas en 2 y 6 áreas respectivamente. El cuestionario comprendió los siguientes módulos: caracterización sociodemográfica, percepción de la discriminación en la sociedad, percepción autorreferente, conocimiento y valoración de la legislación vinculada a la discriminación, conocimiento del INADI, importancia de las acciones del Estado y experiencias propias o presenciadas en torno a la discriminación (INADI, 2013). De este amplio marco de indagación, sólo se toma parte de las experiencias.

Para el caso de Chile se utiliza un módulo de la CASEN, que es una encuesta sociodemográfica de alcance nacional realizada por el Ministerio de Desarrollo Social de ese país, similar a la EPH argentina, y que se viene realizando desde 1990 con una periodicidad bianual o trianual. Según su página web, sus objetivos son:

Conocer periódicamente la situación de los hogares y de la población, especialmente de
aquella en situación de pobreza y de aquellos grupos definidos como prioritarios por
la politica social, con relación a aspectos demográficos, de educación, salud, vivienda,
trabajo e ingresos. En particular, estimar la magnitud de la pobreza y la distribución
del ingreso; identificar carencias y demandas de la población en las áreas señaladas; y
evaluar las distintas brechas que separan a los diferentes segmentos sociales y ámbitos
territoriales.
Evaluar el impacto de la política social: estimar la cobertura, la focalización y la
distribución del gasto fiscal de los principales programas sociales de alcance nacional
entre los hogares, según su nivel de ingreso, para evaluar el impacto de este gasto en el
ingreso de los hogares y en la distribución del mismo. (Casen, 2020).

El marco muestral de la CASEN es construido por jurisdicciones y luego por tres regiones. Distingue entre área urbana -similar al aglomerado urbano-, área rural y resto de área urbana. Los marcos son actualizados y sometidos a procedimientos de simulacro. A los fines de comparabilidad, se trabajará con 166.131 casos, correspondientes a mayores de 18 años. Para ambas encuestas, se utilizan factores de ponderación jurisdiccional (comunal en el caso chileno).

Las técnicas de análisis utilizadas para la comparación son las frecuencias simples y la regresión logística, con dos modelos que se detallarán más adelante. 


\section{Análisis descriptivos}

Para los datos que se utilizarán -la encuesta CASEN 2017 para Chile y la base del Mapa Nacional de la Discriminación en la Argentina- se clasificarán los hogares sobre la base del clima educativo por dominancia, ante las dificultades en poder utilizar un esquema de clases socio-ocupacionales. El clima educativo de los hogares funciona como proxy y, a la vez, como medida sintética, ya que incorpora también la dimensión cultural valorativa (Benner y Kim, 2009; Bernardi y Cebolla, 2014; Fachelli, Molina Derteano y Torrents, 2015; Lain, 2019).

Para realizar la comparación entre ambos se toma la escala ISCED y se agrupa en 4 niveles, cuyas distribuciones se presentan a continuación en la Tabla 1. Como se puede observar, ambos países muestran una distribución similar en los niveles de educación superior, con una leve ventaja para Chile. La diferencia más notoria se encuentra en el caso de los niveles de básica y media: en el caso argentino se concentran más casos en el nivel de básica y media inferior o incompleta con un 43,8\% contra un 34,5\% del país trasandino. Inversamente, en el caso de media completa se concentran más casos en Chile que en la Argentina: $43 \%$ contra un $37,2 \%$.

\section{Tabla 1}

Distribución del clima educativo según máximo logro por dominancia de PSH o cónyuge. Argentina y Chile

\begin{tabular}{l|c|c}
\hline Nivel & Argentina & Chile \\
\hline Hasta ISCED 2: Básica completa y media inferior & 43,8 & 34,5 \\
\hline ISCED 3 y 4 Media completa & 37,2 & 43,0 \\
\hline ISCED 5: Superior no universitario completo & 8,7 & 9,7 \\
\hline ISCED 6 y 7: Superior universitario completo & 10,3 & 12,7 \\
\hline Total & 100,0 & 100,0 \\
\hline
\end{tabular}

Fuente: Elaboración propia sobre la base de CASEN 2017 y Base compilada para el Mapa Nacional de la Discriminación

Para hacer una aproximación a las coordenadas de género, etnia y nacionalidad se toman las variables de sexo, pertenencia a pueblos originarios y origen migratorio de la madre. Respecto a estas últimas es preciso realizar algunas observaciones. Se utiliza la pertenencia a pueblos originarios por un criterio de disponibilidad de datos, pero se asume que es un recorte de una problemática más amplia, que incluye a los afrodescendientes entre otros. A su vez, la encuesta CASEN ofrece una mayor desagregación de etnias de pueblos originarios que en el caso argentino, pero se colapsan en dos categorías para efectos de mayor comparabilidad. Con el mismo criterio, sólo se utiliza la nacionalidad de la madre, por estar presente en ambos relevamientos. 
Tabla 2

Comparación de las variables de sexo, origen migratorio de la madre y pertenencia a pueblo indígena

\begin{tabular}{l|c|c}
\hline Variable & Argentina & Chile \\
\hline Sexo & 48,8 & 46,3 \\
\hline Varón & 51,2 & 53,7 \\
\hline Mujer & 87,4 & 95,3 \\
\hline Origen migratorio madre \\
\hline Nacional & 12,6 & 4,7 \\
\hline Pertenencia a pueblo originario \\
\hline Pertenece & 8,7 & 8,6 \\
\hline No pertenece & 91,3 & 91,4 \\
\hline
\end{tabular}

Fuente: Elaboración propia sobre la base de CASEN 2017 y Base compilada para el mapa nacional de la Discriminación

Los datos muestran una distribución bastante similar para las variables de sexo y pertenencia a pueblo originario, pero en el origen migratorio la proporción de casos argentinos parece ser bastante mayor. Algunos estudios para el caso chileno señalan que el crecimiento de un flujo migratorio hacia el país es un fenómeno relativamente reciente (Mera-Lemp, Bilbao y Martínez-Zelaya, 2020).

\section{Modelo de análisis de regresión}

Puede decirse que tenemos dos modelos que responden a las dos hipótesis que se han ido esbozando. En primera instancia, se propone una hipótesis alternativa que rechace la hipótesis nula de la discriminación como mecanismo de cierre extramercado, basado en los desarrollos de Becker y Arrow. Siguiendo esta lógica, un modelo de regresión logística que se ajuste sólo a la distribución de los hogares sin considerar género, etnia u origen migratorio sería poco o nada significativo, y la diferencia entre los hogares tampoco lo sería. Formulado así:

$\mathrm{H}_{0}$ : La posición de los sujetos en la estructura social no incide en sus chances de ser discriminado/a.

Mientras que dos hipótesis alternativas posibles serían:

$\mathrm{H}_{1}$ : La posición de los sujetos en la estructura social incide en sus chances de ser discriminado/a.

$\mathrm{H}_{2}$ : La posición de los sujetos en la estructura social incide en forma menor en sus chances de ser discriminado/a.

A continuación, se presentan los resultados para los modelos de regresión logística M1 y M2 para la Argentina y Chile. El Modelo 1 sólo incluye la variable de clima educativo, haciendo referencia a la distribución estratificada de los hogares de los encuestados y sus chances de haber sufrido discriminación.

En el Modelo 2, se consideran coordenadas vinculadas a género, etnia y xenofobia. Al agregarse se podría ver cuánto agregan al modelo general y cómo inciden estos factores a su vez. La robustez del M2 servirá, además, para testear en qué medida la hipótesis de discriminación estructural es relativamente débil o fuerte en su capacidad explicativa. 
La Tabla 3 presenta la ejecución del M1 y M2 para la Argentina y la Tabla 4 los presenta para Chile. Una observación interesante es que, en ambos casos, los M1 mejoran considerablemente cuando se les agregan las variables de sexo, pertenencia a pueblos originarios y origen migratorio de la madre. Otro aspecto interesante de la comparación es que los valores de los $\mathrm{R}_{2}$ de Nagelkerke son relativamente similares para ambos modelos, aun cuando las bases de datos tienen orígenes muy disímiles. En el caso argentino se trata de una base de datos resultante de un relevamiento específicamente destinado para la medición de la discriminación, mientras que en el caso chileno es un módulo adosado a una encuesta periódica sociodemográfica. De todos modos, este último es algo más robusto.

Tabla 3

Modelos de regresión M1 y M2 para el caso argentino

\begin{tabular}{l|c|c|c|c}
\hline \multirow{2}{*}{ Variable } & \multicolumn{2}{|c|}{ M1 } & \multicolumn{2}{c}{ M2 } \\
\cline { 2 - 5 } & Sig & Exp (B) & Sig & Exp B \\
\hline Ref: ISCED 6: Superior completo y más & & & & \\
\hline Hasta ISCED 2: Básica completa y media inferior & $0,000^{* *}$ & 1,193 & $0,000^{* *}$ & 1,236 \\
\hline ISCED 3 y 4 Media completa & $0,000^{* *}$ & 1,203 & $0,000^{* *}$ & 1,208 \\
\hline ISCED 5: Superior no universitario completo & $0,000^{* *}$ & 1,083 & $0,000^{* *}$ & 1,157 \\
\hline Sexo: Varón (ref) & & & & \\
\hline Mujer & & & $0,000^{* *}$ & 0,903 \\
\hline Pertenencia a pueblo originario: No pertenece (Ref) & & & & \\
\hline Pertenece a pueblo originario & & & $0,000^{* *}$ & 0,484 \\
\hline Origen migratorio: Madre Nativa (Ref) & & & & \\
\hline Madre extranjera & \multicolumn{2}{|c|}{0,13} & $0,000^{* *}$ & 0,858 \\
\hline R2 de Nagelkerke & \multicolumn{2}{|c}{0,28} \\
\hline
\end{tabular}

Fuente: Elaboración propia sobre la base compilada para el Mapa Nacional de la Discriminación ** Significativo para un $0,05 \%$

Tabla 4

Modelos de regresión M1 y M2 para el caso chileno

\begin{tabular}{|c|c|c|c|c|}
\hline \multirow[t]{2}{*}{ Variable } & \multicolumn{2}{|c|}{ M1 } & \multicolumn{2}{|c|}{ M2 } \\
\hline & Sig & $\operatorname{Exp}(B)$ & Sig & Exp B \\
\hline \multicolumn{5}{|l|}{ Ref: ISCED 6: Superior completo y más } \\
\hline Hasta ISCED 2: Básica completa y media inferior & $0,000^{* *}$ & 0,707 & $0,000^{* *}$ & 0,711 \\
\hline ISCED 3 y 4 Media completa & $0,000^{* *}$ & 0,777 & $0,000^{* *}$ & 0,776 \\
\hline ISCED 5: Superior no universitario completo & $0,000^{* *}$ & 0,934 & $0,072^{* *}$ & 0,942 \\
\hline \multicolumn{5}{|l|}{ Sexo: Varón (ref) } \\
\hline Mujer & & & $0,000^{* * *}$ & 1,465 \\
\hline \multicolumn{5}{|l|}{ Pertenencia a pueblo originario: No pertenece (Ref) } \\
\hline Pertenece a pueblo originario & & & 0,000 & 2,114 \\
\hline \multicolumn{5}{|l|}{ Origen migratorio: Madre Nativa (Ref) } \\
\hline Madre extranjera & & & 0,000 & 1,267 \\
\hline R2 de Nagelkerke & \multicolumn{2}{|c|}{0,17} & \multicolumn{2}{|c|}{0,30} \\
\hline
\end{tabular}




\section{Discusión y comparación}

En primera instancia resulta importante señalar que se trata de un estudio exploratorio destinado a avanzar sobre las bases de comparación en torno a la discriminación estructural y su medición. Retomando nuestro primer objetivo, podemos esbozar una tendencia que se reafirma en el escaso potencial explicativo del M1. Hay significancia pero el abordaje de las prácticas discriminatorias sin precisar los factores de vulnerabilidad asociados al género, etnia o nacionalidad es de carácter limitado.

Un contrapunto interesante es que en el modelo argentino los odd ratio que miden las chances de haber sufrido discriminación contra no haberla sufrida con base en las diferencias en el clima educativo del hogar presentan valores más altos. Una interpretación incorrecta sería que la discriminación por menor capital cultural o por razones socioeconómicas ocurre con mayor frecuencia en la Argentina que en Chile. Los datos no sugieren eso sino que, en la Argentina, la variable que estratifica los hogares con menor capital cultural o de menor potencial socioeconómico incide más porque se trata de una forma de discriminación que se reconoce más claramente. Recordemos que desde un enfoque de discriminaciones múltiples y/o interseccional, el discurso puede privilegiar una forma, sin ser la única. En el caso argentino, los datos del propio Mapa Nacional de la Discriminación mostraban que la discriminación por motivos socioeconómicos era la que registraba mayor frecuencia como discriminación sufrida y como discriminación presenciada (INADI, 2013). Otros estudios de tipo cualitativo arriban a conclusiones similares (Chávez Molina y Molina Derteano, op. cit.; Molina Derteano, op. cit.).

Pero en el caso chileno, ocurre lo inverso e incluso la distinción entre hogares con clima educativo de superior no universitario y superior no universitario se mostró no significativa (aunque por escaso margen). Los hogares con menor capital cultural y/o potencial económico no reconocen haber sufrido más discriminación que aquellos con nivel universitario completo. Y, nuevamente, esto no implica que no estén expuestos a mecanismos de cierre, pero cabría preguntarse si los verían como formas de discriminación.

En este sentido, un modelo de discriminación estructural basado en las posiciones de los hogares como criterio de estratificación en torno a su potencial económico y cultural puede ser significativo, pero tiene un alcance limitado. Se abre la indagación en torno a los valores culturales y su incidencia en la forma de representar la discriminación. ¿Es factible que la sociedad chilena valore más cierto individualismo de mercado y termine por orientar el reconocimiento de sus experiencias en torno a características más "individualizables", como el color de piel o el género? (Barandiarán, op. cit.; Mera-Lemp, Bilbao y Martínez-Zelaya, op. cit.; Montoya, Parrado, Solís y Undurranga et al ., 2020). Este interrogante requiere mayor desarrollo y excede este artículo.

La situación inversa se plantea cuando se consideran las variables de sexo, origen extranjero y pertenencia a pueblos originarios. Allí los valores de los odd ratio son más altos para Chile que para la Argentina, aunque algunas 
observaciones deben hacerse. La primera es que los datos de la Argentina corresponden al año 2013, varios años antes de los movimientos "Ni una menos" o del debate sobre el IVE (Interrupción Voluntaria del Embarazo) que pusieron la agenda feminista y las asimetrías de género en un lugar preponderante (Elizalde y Mateo, 2018; Benente, 2019; Tesoriero, 2020). Los momentos históricos actuales de ambos países señalan en la actualidad una mayor presencia de la agenda feminista y de género.

Sin embargo, el objetivo del modelo era ver si podía probarse un modelo de discriminación estructural sobre la base de la posición socioeconómica más allá de la pertenencia a los grupos considerados como con mayores chances estadísticas de sufrir discriminación por las coordenadas de Patriarcado, Racismo y Xenofobia (Romero, op. cit.).

A partir de la experiencia podría proponerse la réplica en distintos países, siempre sobre la base de la disponibilidad de datos, pero en la que los lineamientos generales podrían ser:

- Análisis descriptivo de la mayor chance de sufrir discriminación y/o presenciarla, con base en criterios de estratificación vinculados con el desarrollo socioeconómico, el bienestar de los hogares, el uso de la escala EGP u otros criterios de estratificación (López Roldán y Fachelli, 2020). Se busca encontrar que haya significatividad.

- Un segundo paso es la inclusión de coordenadas de identificación con grupos sociales cuya identidad sea objeto de subalternidad y/o peligrosidad, y ver en qué medida mejoran las chances y el ajuste del modelo. Inclusive, como fue el caso que aquí mostramos, ver si esas mejoras varían según el país.

- Un tercer paso es un análisis comprensivo con una perspectiva histórica y culturalista y el uso de metodologías cualitativas, que conduzcan a un análisis en profundidad del tránsito del modelo general al de los grupos específicos en cada caso nacional y/o subnacional.

Esta propuesta se encuentra en progreso en estudios que serán difundidos posteriormente. Las piezas se acomodan en el tablero; pero aún falta desarrollar más la partida.

\section{Referencias}

Allen, P. \& Cutts, D. (2019). Women are more likely than men to blame structural factors for women's political under - representation: Evidence from 27 countries. European Journal of Political Research, 58(2), 465-487. doi: https:/ /10.1111/1475-6765.12292

Arrow, K. (1998). What Has Economics to Say about Racial Discrimination? Journal of Economic Perspectives, 12(2), 91-100.

Barandiarán, J. (2012). Researching race in Chile. Latin American Research Review, 47(1), 161-176.

Barbera, M. (2003) Eguaglianza e differenza nella nuova stagione del diritto antidiscriminatorio comunitario. En "Eguaglianza e differenza nella nuova stagione del diritto antidiscriminatorio comunitario", p. 1000-1023. 
Becker, Gary S. (1971). The Economics of Discrimination. Chicago: The University of Chicago Press. Segunda edición. (Primera versión:1957)

Benente, M. (2019). La fuerza de la marea verde inundó transversalmente las casas, las calles. BORDES, 13, 73-81. Recuperado de https://170.210.178.41/OJS/index. $\mathrm{php} /$ bordes/article/download/516/485.

Bernardi, F., y Cebolla, H. (2014). Clase social de origen y rendimiento escolar como predictores de las trayectorias educativas. Revista Española de Investigaciones Sociológicas (REIS), 146(1), 3-21.

Benner, A. D. \& Kim, S. Y. (2009). Intergenerational experiences of discrimination in Chinese American families: Influences of socialization and stress. Journal of Marriage and Family, 71(4), 862-877.

Birzer, M. L. \& Ellis, R. B. (2006). Debunking the myth that all is well in the home of Brown v. Topeka Board of Education: A study of perceived discrimination. Journal of Black Studies, 36(6), 793-814.

Bobo, L. D. \& Fox, C. (2003). Race, racism, and discrimination: Bridging problems, methods, and theory in social psychological research. Social psychology quarterly, 66(4), 319-332.

Browne, I. \& Misra, J. (2003). The intersection of gender and race in the labor market. Annual review of sociology, 29(1), 487-513.

Casen (2020). Descripción y objetivos de la Encuesta. Recuperado de https://www.redatam.org/redchl/mds/casen/WebHelp/informaci_n_casen/ descripci_n_y_objetivos_de_la_encuesta_casen.htm

Cea D'Ancona, M.A. (2005). La exteriorización de la xenofobia. Revista Española de Investigaciones Sociológicas (REIS), 112(1), 197-230.

Cea D’Ancona M.A. (2012). Fundamentos y aplicaciones de la metodología cuantitativa. Madrid: Síntesis.

Cea D'Ancona, M. A. \& Valles Martínez, M. S. (2015). Evolución del racismo, la xenofobia $y$ otras formas conexas de intolerancia en España (Informe-Encuesta 2014). Ministerio de Empleo y Seguridad Social. Recuperado de https://tandis.o dihr.pl/bitstream/20.500.12389/22212/1/08478spa.pdf.

Cohen, N. (2009). No sólo es cuestión de migrantes: migraciones externas y exclusión social. En S. Goinheix (Comp.), Conflictos y expresiones de la desigualdad y la exclusión en América Latina (pp. 35-79). Buenos Aires: El Aleph.

Elizalde, S. y Mateo, N. (2018). Las jóvenes: entre la "marea verde" y la decisión de abortar. Salud colectiva, 14, 433-446. Recuperado de https://www.scielosp.org/a rticle/scol/2018.v14n3/433-446/es/.

Fachelli, S., Molina Derteano, P. y Torrents, D. (2015). Un análisis comparado de las desigualdades de acceso a la universidad en Argentina, España y México en 2013. Revista de Educación y Derecho 15, 1-27.

Grimson, A. (1997). Relatos de la diferencia y la igualdad. Los bolivianos en Buenos Aires. Nueva sociedad, 147, 96-107.

Grimson, A. (2006). Nuevas xenofobias, nuevas políticas étnicas en la Argentina. Migraciones regionales hacia la Argentina. Diferencia, desigualdad y derechos, 69-97.

Grimson, A. , Ng, G. y Denardi, L. (2016). Las organizaciones de inmigrantes chinos en Argentina. Migración y desarrollo, 14(26), 25-73.

INADI (Instituto Nacional contra la Discriminación, la Xenofobia y el Racismo) (2013). Mapa nacional de la discriminación. Buenos Aires: INADI. 
Kaufman, A. (2010). Dignus inter pares. Un análisis comparado del derecho antidiscriminatorio. Buenos Aires: Abeledo-Perrot.

Kerwin, K. C. \& Guryan, J. (2007). Prejudice and the Economics of Discrimination. NBER Working Paper Series, 13661.

Lain, J. (2019). Discrimination in a search and matching model with self-employment. IZAJournal of Development and Migration, 9(1), 6.

López-Roldán, P. \& Fachelli, S. (2020). A General Model for the Comparative Analysis of Social Inequalities Between Europe and Latin America. En Towards a Comparative Analysis of Social Inequalities between Europe and Latin America (pp. 3-34). Cham: Springer.

Margulis, M. (1999). La racialización de las relaciones de clase. En Margulis, M., Urresti, M. et al. La segregación negada. Cultura y discriminación social, Buenos Aires: Biblos. pp 34-56

Mera-Lemp, M. J., Bilbao, M. y Martínez-Zelaya, G. (2020). Discriminación, aculturación y bienestar psicológico en inmigrantes latinoamericanos en Chile. Revista de Psicología, 29(1). Recuperado de https://revistateoria.uchile.cl/index. $\mathrm{php} / \mathrm{RDP} /$ article/view/55711

Molina Derteano, P. (2019). Variables estructurales y discriminación: el rol de la escolarización y el origen social., Ponencia en el V Seminario Internacional Desigualdad y Movilidad Social en América Latina, Santiago de Chile, 6 al 8 de Mayo de 2019.

Molina Derteano, P. (en prensa). "Discriminación estructural: los espejos de la desigualdad en preadolescentes y adolescentes". En Molina Derteano, P. y Chávez Molina, E. (Comps.). Diversidad en la desigualdad. Debates teóricos y exploraciones empiricas recientes.

Montes Berges, B. (2008). Discriminación, prejuicio, estereotipos: conceptos fundamentales, historia de su estudio y el sexismo como nueva forma de prejuicio. Revista Iniciación a la Investigación, (3)45, 1-16.

Montoya, A. M., Parrado, E., Solís, A. y Undurraga, R. (2020). Discriminación de género en el mercado de créditos de consumo en Chile. Serie Políticas Públicas y Transformación Productiva $\mathrm{N}^{\circ} 34$,

MSSI (Ministerio de Salud, Servicios Sociales e Igualdad) (2012). Estudio anual sobre la discriminación por el origen racial o étnico: la percepción de las potenciales victimas, 2011. Madrid: MSSI.

Nuvaez Castillo, J. J. (2019). Alcance sociocultural de la discriminación laboral en razón del género y la edad. Iustitia Socialis: Revista Arbitrada de Ciencias Jurídicasy Criminalisticas, 4(6), 4-27. doi: http://dx.doi.org/10.35381/racji.v4i6. 286 Recuperado de https://dialnet.unirioja.es/servlet/articulo?codigo $=7049440$

Pincus, F. (1996). Discrimination comes in many forms: Individual, institutional, and structural. The American Behavioral Scientist, Nov/Dec 1996, 40(2). pp 186-194

Reskin, B. (2012). The race discrimination system. Annual Review of Sociology, 38, $17-35$.

Romero, M. (2017). Introducing intersectionality. Cambridge: John Wiley \& Sons.

Solís, P. (2017). Discriminación estructural y desigualdad social. Recuperado de http:/ /repositorio.dpe.gob.ec/handle/39000/2084

Solís, P., Krozer, A., Batista, C. A. y Graniel, B. G. (2019). Discriminación étnico-racial en México: una taxonomía de las prácticas. CÁTEDRA UNESCO, 55.

Tesoriero, V. (2020). La Marea Verde como nuevo actor político. Cambios en el movimiento feminista argentino. PLAZA PÚBLICA. Revista de Trabajo Social, 
22, 101-107. Recuperado de https://ojs2.fch.unicen.edu.ar/ojs-3.1.0/index.php/ plaza-publica/article/view/691.

Wagman, D. (2003). Metodología y herramientas para el diagnóstico de la discriminación. II Jornadas Estatales Empleo e Inmigración. Estrategias de Comunicación para la Promoción de la Igualdad de Trato, 12.

Wieviorka, M. (2007). La mutación del racismo. Revista mexicana de ciencias políticas $y$ sociales, 49(200), 13-23. 